\title{
Chlamydial pneumonia in the low birthweight neonate
}

\author{
A A ATTENBURROW AND C M BARKER \\ Special Care Baby Unit, Derby City Hospital and Department of Virology, Derbyshire Royal Infirmary
}

SUMMARY Five low birthweight neonates who developed a severe pneumonia are described. Chlamydia trachomatis was isolated from the respiratory tract of all infants. They had protracted respiratory problems despite eradication of the organism by chemotherapy.

Chlamydial pneumonia was first described in $1975 .^{1}$ It is characterised by the onset of a chronic, afebrile pneumonia at 2 to 8 weeks of age, not necessarily preceded by conjunctivitis, and is associated with a peripheral eosinophilia; raised immunoglobulins $\mathbf{M}$, $\mathrm{G}$, and $\mathrm{A}$; and raised titres to Chlamydia trachomatis. Bilateral, symmetrical, interstitial infiltrates and hyperinflation are visible on chest radiographs. ${ }^{2}$ Although maternal genital chlamydial infection predisposes to preterm delivery, ${ }^{3}$ severe pneumonia in a very low birthweight infant has only recently been described, on that occasion with fatal outcome. ${ }^{4}$ We have now investigated a total of five patients.

\section{Materials and methods}

C trachomatis was isolated by culture. The following method is used at Derbyshire Royal Infirmary (for cases 1, 3, 4, and 5). The growth medium is Eagles minimum essential medium, containing $10 \%$ fetal calf serum; $4 \mathrm{ml}, 4.4 \%$ sodium bicarbonate solution, and antibiotics (streptomycin $100 \mu \mathrm{g} / \mathrm{ml}$, vancomycin $100 \mu \mathrm{g} / \mathrm{ml}$, amphotericin $5 \mu \mathrm{g} / \mathrm{ml}$ ). The cells (HEP 2 or McCoy, or both) are seeded at 175000 cells $/ \mathrm{ml}$ onto $12 \mathrm{~mm}$ diameter coverslips in screw capped plastic tubes. These are incubated at $37^{\circ} \mathrm{C}$ until the cells are confluent (approximately six to 12 hours). About $0.5 \mathrm{ml}$ of the specimen is inoculated per tube, and centrifuged at $5000 \mathrm{~g}$ for one hour at $35^{\circ} \mathrm{C}$. These tubes are incubated at $37^{\circ} \mathrm{C}$ for two hours before being media-changed to growth medium (as above) containing $530 \mathrm{mg}$ glucose and $100 \mu \mathrm{g}$ cycloheximide. Further incubation for 60 hours at $37^{\circ} \mathrm{C}$ occurs before fixation in methyl alcohol and staining with $10 \%$ Giemsa.

Chlamydial serology was performed at the Insti- tute of Ophthalmology, London, by microimmunofluorescence.

Case 1. A boy was born at 26 weeks' gestation by spontaneous vaginal delivery to an 18 year old unmarried mother. His birthweight was $830 \mathrm{~g}$. He was in good condition at birth, but from 10 hours of age he required intermittent positive pressure ventilation for recurrent apnoea, and intravenous penicillin and gentamicin were given after an infection screen. Mild idiopathic respiratory distress syndrome was diagnosed from the first chest radiograph, but on the second day there was a rather more floccular pattern in both lung fields. Bacterial cultures were persistently negative, but $C$ trachomatis was isolated from tracheal secretion obtained on day 11. Chlamydial serology of paired sera on days 11 and 18 was negative. Viral culture and serology were not performed. Despite treatment with erythromycin $(80 \mathrm{mg} / \mathrm{kg}$ per day), lung function continued to deteriorate. From day 21 , sulphadimidine was started $(160 \mathrm{mg} / \mathrm{kg}$ per day $)$, and finally from day 38 intramuscular oxytetracycline $(7.5$ $\mathrm{mg} / \mathrm{kg}$ per day) was given. This produced a temporary improvement in radiological appearance and a reduction in oxygen requirements. He continued to deteriorate, despite negative bacterial and chlamydial cultures, with increasing ventilatory requirements and opacification on chest radiograph, and died at the age of 42 days. Permission to conduct a necropsy was refused. $C$ trachomatis was not isolated from the mother's endocervical swab taken 14 days after delivery.

Case 2. A girl was born at 32 weeks' gestation to a 31 year old mother (para 6) who had received no 
antenatal care. She was delivered by caesarean section. Her birthweight was $1560 \mathrm{~g}$. She was in good condition at birth but soon developed respiratory distress requiring ventilation. After an infection screen, she was started on intravenous penicillin and gentamicin. Her initial chest radiograph confirmed severe idiopathic respiratory distress syndrome, and during the next two days she developed bilateral pneumothoraces which were successfully drained. There was severe pulmonary interstitial emphysema.

Ventilation was stopped after eight days. She remained tachypnoeic, requiring oxygen treatment, and there were bilateral crepitations on auscultation of her chest. Antibiotic treatment was stopped after 10 days. Chest radiographs showed ill defined opacification in the upper and lower zones. Bacterial cultures were persistently negative. On day 20 , endotracheal secretions obtained under direct vision yielded an isolate of $C$ trachomatis. She was treated with intravenous erythromycin $(50 \mathrm{mg} / \mathrm{kg}$ per day) and with co-trimoxazole $(125 \mathrm{mg} / \mathrm{kg}$ per day, expressed as a dosage of sulphamethoxazole). She had a total of 22 days erythromycin and 10 days co-trimoxazole treatment, with no improvement, although chlamydia was no longer detectable by culture of respiratory tract secretions. Chlamydial titres were not done. She required oxygen treatment until the 75th day of life. Repeated chest radiographs showed changes compatible with bronchopulmonary dysplasia. She was discharged home age 81 days having grown well.

Chlamydial cultures of the mother's endocervical swab were negative three weeks post partum.

Case 3. A girl, the first of twins conceived after artificial insemination by donor, was born at 25 weeks' gestation by spontaneous breech delivery to a 38 year old primiparous mother. The membranes had been ruptured for 12 to 13 days. Her mother had had a vaginal discharge throughout most of the pregnancy, and was being treated with amoxycillin and metronidazole. The liquor was purulent. The infant's birthweight was $750 \mathrm{~g}$. She was asphyxiated at birth and required resuscitation with oxygen via bag and mask. Ventilation was instituted shortly after delivery while she was being transferred from another unit, and was continued because of worsening respiratory distress. The initial chest radiograph was clear. She was given intravenous penicillin and gentamicin, because of the foul liquor and appreciable neutrophilia $\left(25 \cdot 6 \times 10^{9} / 1\right)$. Bacterial cultures, however, were negative.

A chest radiograph at age 8 days showed diffuse right sided consolidation. C trachomatis was cultured from tracheal secretions. While these results were awaited, she was treated with intravenous cefotaxime, but this was changed to intravenous erythromycin at a dosage of $65 \mathrm{mg} / \mathrm{kg}$ per day on day 11. As she did not improve, co-trimoxazole was added on day 13 at a dose of $80 \mathrm{mg}$ sulphamethoxazole/kg per day. After 10 days intravenous oxytetracycline $(8 \mathrm{mg} / \mathrm{kg}$ per day) was substituted as deterioration continued. She was given a total of 24 days erythromycin, 34 days co-trimoxazole, and 12 days oxytetracycline treatment. Chlamydial cultures of tracheal secretions became negative. Chlamydial serology was negative at 2 and 7 weeks and 6 months of age. Serology for cytomegalovirus, herpes viruses types 1 and 2, and Pneumocystis carinii were negative. Ipratropium bromide was instilled via the endotracheal tube in an attempt to reduce secretions. At this stage her ventilatory requirements began to fall and ventilation was stopped on day 45 . She continues to do well, and has no residual changes on chest radiographs.

Chlamydial culture of the donor semen and the maternal endocervical swab (approximately two weeks post partum) were negative.

Case 4. A boy, the second twin of the patient in case 3 , was born by spontaneous breech delivery. He waß in his own unruptured amniotic sac. His birthweighe was $800 \mathrm{~g}$ and he was in good condition at birth. He्हु was electively intubated and ventilated for transfe between units, and was extubated at 21 hours of age. In view of the purulent liquor in his sister's amniotic sac, he was treated with intravenous penicillin and gentamicin. From age 3 days he suffered from recurrent episodes of bradycardia, and was treated with intravenous aminophylline. Antibiotic treatment was stopped as bacterial cultures were negative.

On day 11 , he deteriorated. He had a raised neutrophil count of $27 \cdot 5 \times 10^{9} / 1$. After an infection screen was performed, he was started on intravenous erythromycin $(65 \mathrm{mg} / \mathrm{kg}$ per day) and ampicillin. The ampicillin was stopped after three days. From day 14, he needed ventilation because of increasingly severe episodes of bradycardia. On day 22 , despite continuing erythromycin treatment, both C trachomatis and Escherichia coli were grown from tracheal secretions. Co-trimoxazole, at a dosage of $80 \mathrm{mg}$ sulphamethoxazole/kg per day was added intravenously, and oxytetracycline $8 \mathrm{mg} / \mathrm{kg}$ per day was substituted for erythromycin. The chest radiograph showed persisting changes in the right lower zone. Chlamydial cultures became negative. Chlamydial serology at two weeks was negative, by seven weeks there was an IgG titre of 1 in 16 to serovars $\mathrm{A}$ to $\mathrm{C}$, and by six months there was both an IgG and IgM response of 1 in 16 to these serovars. 
Serology for cytomegalovirus, herpes viruses type 1 and 2 , and pneumocystis were negative.

Ipratropium bromide was instilled via the endotracheal tube in an attempt to reduce his profuse secretions. From day 34 , his ventilatory requirements decreased. His antibiotics were stopped after a total of 12 days' treatment with tetracycline and 15 days' with co-trimoxazole. He was successfully extubated on day 48 . His chest radiograph film was clear and oxygen treatment was stopped on day 50.

Nine days later, he had increasing bradycardia and stridor, with consolidation along the right heart border of the chest radiograph. He required a further seven days' ventilation and was given intravenous penicillin and gentamicin during that time. Both bacterial and chlamydial cultures were negative.

He has persistent upper airways obstruction which has necessitated a tracheostomy. His chest radiograph is again clear.

Case 5. A boy, the first child of an unmarried, 19 year old black mother, was born at 31 weeks' gestation by caesarean section. His birthweight was $1240 \mathrm{~g}$. His mother had been noted to have a cervical erosion at 29 weeks' gestation, and a high vaginal swab at that time had cultured Gardnerella. She had been treated with oral metronidazole. There was no prolonged rupture of membranes. C trachomatis was isolated from eyes, throat, and nasopharynx at birth. This organism was also cultured from the maternal endocervical swab.

He required ventilation from the age of 3 hours because of increasing respiratory distress. Initial radiographic appearances were those of hyaline membrane disease. Penicillin and gentamicin were begun shortly after birth. Erythromycin $(80 \mathrm{mg} / \mathrm{kg}$ per day) was begun on day 4 once the initial cultures were available, along with flucloxacillin as Staphylococcus epidermidis was grown on blood culture. On day 5, there were still positive cultures of $C$ trachomatis from eyes, throat, and tracheal secretions. Ventilation was stopped after five days, but he remained dependent on oxygen until day 49 . On day 11, because of continuing respiratory distress and right sided consolidation on chest radiograph, co-trimoxazole was begun at a dosage of $80 \mathrm{mg}$ sulphamethoxazole/kg per 24 hours, and was continued until day 55 , the dose being halved on day 48. Bacterial cultures from the respiratory tract were persistently negative and chlamydial cultures were negative from day 12 .

His clinical course was complicated by a symptomatic persistent ductus arteriosus, which was treated with fluid restriction, regular frusemide, and a course of intravenous indomethacin.
Chlamydial titres were negative at 6 weeks but at 11 weeks there were IgG titres of 1 in 16 to serotypes $A$ to $C$ and $D$ to $K$. There was no rise in IgM. Serology for cytomegalovirus and pneumocystis were negative but there was an IgG titre of 1 in 16 at weeks 6 and 11 to herpes virus type $I$.

\section{Discussion}

These neonates all developed a pneumonia associated with isolation of $C$ trachomatis from their tracheal secretions. In two of them, we have documented a low and late rise in antibody titres, rather reminiscent of that described in the immunocompromised adult. ${ }^{6}$ If this is indeed a chlamydial pneumonia, it seems to have somewhat different characteristics in the low birthweight neonate compared with the larger infant. It is a more severe illness, requiring prolonged ventilation, and lasting up to 42 days. The age of onset is slightly earlier. The radiographic changes are variable and not always symmetrical. The blood picture showed a neutrophilia in two cases rather than an eosinophilia.

Previously recommended treatment has been a 14 to 21 day course of either erythromycin or a sulphonamide, clinical improvement occurring at a median of four days after treatment. ${ }^{5}$ These patients did not improve on either erythromycin or sulphonamides, and in fact one (case 4) developed chlamydial pneumonia while being treated with erythromycin. In three patients, treatment with intravenous oxytetracycline, which is often used in adult chlamydial infection but is not recommended in childhood as it causes discoloration of the teeth and enamel hypoplasia, ${ }^{7}$ was felt to be justified. This seemed to be related to a clinical improvement in all three patients, albeit only temporarily in one.

Two patients developed bronchopulmonary dysplasia. Radkowski and co-authors have discussed the radiological similarities, and have tried to link the two conditions. ${ }^{8}$ These two patients had higher ventilation requirements than the others, however, one due to severe idiopathic respiratory distress syndrome and the other related to the severe pneumonitis. It is possible that this predisposed to the bronchopulmonary dysplasia rather than chlamydial infection.

It is difficult to draw conclusions and recommendations from only five cases. Any neonate, however, who has protracted respiratory problems, with radiographic changes suggestive of infection, and negative bacterial cultures should have tracheal secretions obtained for chlamydial culture. If positive, an initial course of two weeks' erythromycin and a sulphonamide should be considered. If no 
improvement occurs and the infant still requires ventilation then a further two weeks of oxytetracycline treatment may be justifiable.

We thank Dr K L Dodd for permission to discuss four of his patients and for advice and support and Dr D Bullock for help. We also thank Dr L Alroomi for allowing us to include one of her patients in this study (case 2).

\section{References}

1 Schachter J, Lum L, Gooding CA, Ostler B. Pneumonitis following inclusion blennorrhoea. J Pediatr 1975;87:779-80.

2 Tipple MA, Beem MO, Saxon EM. Clinical characteristics of the afebrile pneumonia associated with Chlamydia trachomatis infection in infants less than 6 months of age. Pediatrics 1979;63:192-7.

${ }^{3}$ Martin DH, Kaitsky L, Eschenbach DA, et al. Prematurity and perinatal mortality in pregnancies complicated by maternal Chlamydia trachomatis infections. JAMA 1982;247:1585-8.

${ }^{4}$ Attenburrow AA. Infant chlamydial pneumonia (letter). $\mathrm{Br} \overrightarrow{\overrightarrow{\mathrm{B}}}$ Med J 1983;ii:1219-20.

${ }^{5}$ Beem MO, Saxon E, Tipple MA. Treatment of chlamydial pneumonia of infancy. Pediatrics 1979;63:198-203.

6 Ito JI, Comess KA, Alexander ER, et al. Pneumonia due to Chlamydia trachomatis in an immunocompromised adult. N Engl J Med 1982;307:95-8.

7 Pindborg JJ. Disturbances in tooth formation: aetiology. In: Pindborg JJ, ed. Pathology of the dental hard tissues. Copenhagen: Scandinavian University Books, 1970;167-71.

's Radkowski MA, Kranzler JK, Beem MO, Tipple MA. Chlamydial pneumonia in infants. Radiography in 125 cases. AJR 1981;137:703-6.

Correspondence to Dr A A Attenburrow, Department of Neonatal Medicine and Surgery, City Hospital, Nottingham.

Received 8 July 1985 